\title{
NGO Corner
}

\section{Youtube-Programm für NGOs}

Bisher war das Nonprofit-Programm von YouTube nur für in Australien, Großbritannien und Nordirland, Irland, Kanada und USA ansässige Organisationen nutzbar. Seit Mai ist das kostenlose Programm, welches NGOs zusätzliche Funktionen für deren Youtube-Kanal ermöglicht, nun auch in Deutschland verfügbar. Im Wesentlichen beinhaltet es fünf Funktionen. Erstens die Option Call-to-Action-Overlays, mit einem Link zu einer externen Webseite in die Videos zu integrieren. So kann etwa im Video dazu aufgefordert werden eine Petition zu unterschreiben und der Button führt direkt zur entsprechenden Seite. Diese Links konnte man bis jetzt nur in den begleitenden Informationstext einfügen; der allerdings entfiel, wenn das Video auf einer anderen Seite eingebettet wurde. Darüber hinaus kann man einen Spendenbutton direkt unter dem Video einbinden. Hierfür greift Youtube auf die in Deutschland kaum verbreitete Funktion GoogleWallet zurück. Die vierte zusätzliche Funktion sind externe VideoAnmerkungen und auch die Option des Live-Streamings ist neu. Insgesamt ergeben sich für den eigenen Youtube-Kanal neue Gestaltungsmöglichkeiten. So können das Logo und die Farben der Organisation eingebunden werden. Voraussetzung für die Teilnahme am Programm ist, dass die eigene Organisation in Deutschland als gemeinnützig anerkannt und bei Google mit einem eigenen Account registriert ist.

\section{Neue EU-Plattform der Zivilgesellschaft zur Bekämpfung des Menschenhandels}

Ende Mai haben sich mehr als 100 europäische zivilgesellschaftliche Organisationen in der von der EU Kommission eingerichteten neuen EU-Plattform der Zivilgesellschaft zur Bekämpfung des Menschenhandels zusammengeschlossen. Die Plattform soll Organisationen, die in den Bereichen Menschenrechte, Rechte des Kindes, der Frau, der Migranten sowie der Gleichstellung der Geschlechter und des Opferschutzes auf lokaler, nationaler und EU-Ebene tätig sind, als Forum dienen ihre Kräfte zu bündeln, in dem sie ihre Erfahrungen und Vorstellungen austauschen sowie ihre Netzwerke ausbauen. Die Kommission will die Plattform durch die Organisation regelmäßiger Mitgliedertreffen unterstützen. Das nächste Treffen ist für den Herbst geplant.

\section{Zivilgesellschaftliche Zusammenarbeit - Partnerschaft für eine menschenrechtsbasierte nachhaltige Entwicklung}

Die Fraktion Bündnis 90/Die Grünen kritisiert eine aus ihrer Sicht bestehende „Gängelung der deutschen Zivilgesellschaft“ in der Entwicklungszusammenarbeit. In ihrem Antrag (17/13728) vom 06. Juni wendet sie sich gegen eine zunehmende Einflussnahme des BMZ gegenüber NGOs, etwa durch die Kontrolle regierungs- und unternehmenskritischer Publikationen. Ebenso ist die neue Designrichtlinie des BMZ Teil der Kritik. Außerdem soll der Aufbau von Parallelstrukturen zu den Aktivitäten von NGOs durch die beim BMZ angesiedelte »Servicestelle Engagement Global« beendet werden. Die
Abgeordneten forderten weiter eine konsequentere Linie gegenüber Partnerländern, in welchen die Arbeit von NGOs massiv eingeschränkt wird. Die Entwicklungszusammenarbeit mit diesen Ländern solle einer kritischen Prüfung unterzogen werden.

http://dip21.bundestag.de/dip21/btd/17/137/1713728.pdf

\section{Forderungskatalog der Zivilgesellschaft an die Wissenschafts- und Forschungspolitik}

Ein breites überparteiliches Bündnis von zivilgesellschaftlichen Organisationen hat am 31. Mai einen Zehn-PunkteKatalog für mehr Transparenz und Nachhaltigkeit in der Wissenschafts- und Forschungspolitik in Deutschland vorgelegt. Konkret zielen Die Forderungen darauf ab: die Transparenz wissenschaftspolitischer Entscheidungen durch Einbeziehung der Zivilgesellschaft und den freien Zugang zu Forschungsergebnissen zu erhöhen, die Kompetenzen der Wissenschaft stärker für die Bewältigung drängender Themen wie Klimaschutz oder den Erhalt von Biodiversität, nachhaltiges Wirtschaften, globale Gerechtigkeit oder eine humane Arbeitswelt zu nutzen, und dafür die inter- und transdisziplinäre Nachhaltigkeitsforschung im Wissenschaftssystem zu stärken. Bis Mai wurden die „Zivilgesellschaftlichen Forderungen an die Wissenschafts- und Forschungspolitik " von siebzehn Organisationen unterzeichnet. Es beteiligten sich u.a. Umweltverbände, Entwicklungshilfe- und Gesundheitsorganisationen und Kirchen. Das Bündnis ist offen für weitere Akteure und will sich in Zukunft aktiv an der wissenschaftspolitischen Debatte beteiligen.

http:/www.dnr.de/downloads/zivilgesellschaftliche-forderungen-wissenschaft.pdf

\section{Neuer Leitfaden Internet für NGOs}

Das Internet verändert die Arbeit und die Kommunikation von NGOs. Der im Mai erschienene neue Leitfaden "Internet für NGOs" der Agentur Wigwam und des betterplace lab_widmet sich den verschiedenen Dimensionen dieses Wandels. Insgesamt 34 Autoren befassen sich sehr praxisbezogen mit einer Vielzahl von relevanten Aspekten der digitalen NGO-Kommunikation bzw. des NGO-Campaigning. Die Themenblöcke des Leitfadens sind "Online arbeiten und organisieren", "Online Spenden sammeln", “Online kommunizieren”, "Online Menschen mobilisieren” und "Das Online von morgen”. Neben zahlreichen konkreten Praxisbeispielen, finden sich Überblicksartikel, Interviews sowie Artikel die sich mit zu den verschiedenen Zwecken einsetzbare Online-Tools befassen und Checklisten, etwa zum Einsatz von Social Media in Kampagnen. Der komplette Leitfaden ist kostenlos im Netz verfügbar.

http://www.ngoleitfaden.org/ 